\title{
Facile preparation of superhydrophobic metal foam for durable and high efficient continuous oil-water separation
}

Yue Hu, Yanji Zhu, Huaiyuan Wang*, Chijia Wang, Hongwei Li, Xiguang Zhang, Ruixia Yuan, Yiming Zhao

College of Chemistry and Chemical Engineering, Northeast Petroleum University, Daqing, China ABSTRACT: Foam-based material with unique wettability is one of the promising materials for developing oil-spill cleanup, benefiting from its intrinsic interconnected three-dimensional skeleton, oil permeation and water repellency. However, obtaining a foam-based material capable of in situ separating oil spills, especially with superhydrophobic durability and simple recycling mode, remains challenging. Herein, we reported a one-step spraying approach to fabricate fluorinated ethylene propylene (FEP)/ polyvinylidene fluoride (PVDF)/ ultrafine polyurethane (UPU)/ hydrophobic nanosilica $\left(\mathrm{HSiO}_{2}\right)$-decorated nickel foam coating with a static water contact angle (WCA) of $157 \pm 1.5^{\circ}$ and a static oil contact angle (OCA) of $0^{\circ}$. Owing to the foldability of the coated nickel foam, we established a continuous in-situ collection apparatus by external pumping on 3D nickel foam box. UPU addition endowed the resultant 3D nickel foam with excellent interfacial adhesion. After 7 days of continuous oil-water experiment that simulated cleanup oil-spill, the static WCA of the 3D nickel foam with $10 \mathrm{wt} \%$ UPU was still above $130^{\circ}$. Notablely, after heat treatment at $240{ }^{\circ} \mathrm{C}$, the fabricated $3 \mathrm{D}$ nickel foam coating could be refreshed from the damaged surface to superhydrophobic.

Keywords: Continuous oil-water separation; High efficiency; Superhydrophobic durability; One-step spraying;

${ }^{*}$ Corresponding author. Tel.: 0086-4596503083; Fax: 0086-4596503083.

E-mail address: wanghyjiji@163.com (H.Y.Wang) 


\section{Introduction}

Oil/water separation has always been focused by the scientist and engineer owing to the rapid development of petroleum industry accompanied with the ever-increasing oil spillages and oily wastewater [1-3]. Some conventional approaches to deal with oil/water mixtures such as oil skimmers, centrifuges and controlled burning [4-6], have the drawbacks of time-consuming, inefficiency, high-energy consuming or environmental unfriendly. Thus, efficient oil/water separation is still full of challenge, addressing this challenge urgently requires to develop a new functional material that can effectively remove oil from water.

Currently, hydrophobic and oleophilic material is relatively promising in oil-water separation because of its intrinsic different wettability of oil and water [7,8]. Based on this, numerous hydrophobic porous materials synthesis routes (including dip-coating $[9,10]$, vapor deposition [11-13], electrochemical deposition [14-16], in-situ synthesis [17-21], electrospinning [22,23]) have been reported to fabricate superhydrophobic and superoleophilic surface. Besides, spraying technique is a one-step process to obtain a special wetting coating occupying appropriate surface roughness and chemical composition [24,25]. Recently, the spraying fabrication of porous functionalized membranes have been applied in oil-water separation. Gondal et al. [26] designed a stainless steel mesh incorporated with nanostructured $\mathrm{TiO}_{2}$, which provided both micro and nanoscale structure. The fabrication of polyelectrolyte-fluorosurfactant complexes/SiO${ }_{2}$-coated mesh films exhibited a much improved superoleophobicity both in air and underwater [27]. Those researches have obtained the homogeneous and stable film by spraying, therefore, designing a stable superhydrophobic and superoleophilic material that skim the floating oil droplets is 
attemptable by spraying technology for marine oil spillage remediation and petroleum exploitation treatment.

Polyurethane (PU) sponge as one of the most versatile materials, has been widely used in oil/water separation aspect [28-30]. In recent years, special attention is paid to superhydrophobic and superoleophilic PU sponge continuously separating oil in situ from water through a variety of techniques, because sponge is porous material used in rapidly removing oils from wastewater with high selective absorption. Crater-like functionalized graphene layers were formed on the polyurethane sponge surface, then in conjunction with a vacuum system to continuously separate oil/water mixtures [31]. Zhang and coworkers applied modified silica nanoparticles to attach firmly onto the surface of PU sponge silica for absorbing oils from water surface, the $\mathrm{SiO}_{2}$ gave rise to the nano-scaled roughness [32]. Although these researches have demonstrated that PU sponge is a good candidate to be used for continuously oil cleanup operation, it is difficult to support the weight of the adsorbed oil due to the limitation of low strength of sponge. The absorption efficiency would inevitably decrease after scaling-up [20,33]. Meanwhile, few superhydrophobic PU coatings were reported in literature to be applied in oil-water separation [24]. The above-mentioned facts inspire us to utilize metal foam with sponge-like structure covered PU to construct functional coating.

In this study, we report that the superhydrophobicity and superoleophilicity composite coatings can be facilely fabricated on nickel foam by simply spraying the etched nickel foam with a mixed solution containing fluorinated ethylene propylene (FEP), polyvinylidene fluoride (PVDF), ultrafine polyurethane (UPU) and hydrophobic silica nanoparticles $\left(\mathrm{HSiO}_{2}\right)$ (defined as FPUH coated nickel foam) (Scheme 1). The FPUH coated nickel foam synergistically enabled a fluorine-covered surface to be formed and a firm connection structure to be constructed, both enhancing substantially 
hydrophobic stability. The as-prepared nickel foam shows outstanding properties of superhydrophobic durability, high efficiency, high separation speed, resistance against corrosion and reproducibility. Afterward, the prepared nickel foams can be simply folded, and then covered the stainless steel skeleton to form a hollow box, which combines the functions of oil boom and oil skimmer vessel. A boat is surrounded by many fabricated hollow boxes with pumps for remediating crude oil spills, which ensures that this promising oil-water separation system can be applied for practical oil spill treatment.

\section{Experimental section}

\subsection{Materials}

Nickel foam (volume density: $0.32 \mathrm{gcm}^{-3}$, average pore diameter: $130 \mu \mathrm{m}$, pore number: $110 \mathrm{PPI}$ ) were obtained from Taiyuan Lizhiyuan Material Technology Co. Ltd. (China). Polyurethane (PU) were bought from Ningbo Hanma Co. Ltd. (China). Fluoride (PVDF, FR904, Mw =380,000 g/mol, $\mathrm{d}=25 \mu \mathrm{m}$ ) were obtained from Shanghai 3F Co. Ltd. (China). Fluorinated Ethylene Propylene (FEP, $\mathrm{d}=6.5 \mu \mathrm{m})$ were obtained from DuPont, USA. Hydrophobic silica nanoparticles $\left(\mathrm{HSiO}_{2}\right)$ were purchased from Hi-Tech Reagent Factory of Nanjing in China. Oils (lubricating oil, silicon oil, petroleum ether and corn oil) and organic solvents (dichloromethane, chloroform and toluene) were supplied by Huadong Reagent Factory, Shenyang, China. Ethanol, distilled water and sal communis were used throughout the experiments.

\subsection{Preparation of superhydrophobic nickel foam}

Under the conditions of $6 \mathrm{~h}$ high energy ball milling, the ultrafine polyurethane powder (UPU) were prepared. Firstly, the nickel foam (110 PPI) was cut into pieces according to the model (Fig. S1a), and it was ultrasonically etched with $25 \mathrm{~mL}$ hydrochloric acid and $25 \mathrm{~mL}$ distilled water for 
$10 \mathrm{~min}$. Next, $0.1 \mathrm{~g}$ Hydrophobic $\mathrm{HSiO}_{2}$ nanoparticles was added to $25 \mathrm{~mL}$ absolute alcohol and the solution was stirred for $30 \mathrm{~min}$, after which a certain amount of UPU, the same amount of PVDF and FEP were added, and then dispersed by ultrasound for $10 \mathrm{~min}$ (the $\mathrm{FEP} / \mathrm{PVDF} / \mathrm{HSiO}_{2}$-decorated nickel foam named as FPH coated nickel foam). Furthermore, the nickel foams were made by spraying methods where the mixtures were placed into spray guns and spraying with a laboratory scale air pump. The functional coatings were fabricated by a spray gun pressure of 6 bar and a spraying distance of $15 \mathrm{~cm}$ between the spray gun and the nickel foam. Finally, the nickel foams were calcined at $240{ }^{\circ} \mathrm{C}$ for $90 \mathrm{~min}$.

\subsection{Separation experiments}

Firstly, we folded the fabricated nickel foam to make a closed hollow box with the size of $3 \times 3 \times$ $3 \mathrm{~cm}$ (Fig. S1a and S2). As exemplified in Fig. S1b, the three-dimensional FPUH coated nickel foam (3D nickel foam) connected pipes and peristaltic pump was used as a continuous self-controlled oil collection system, and then the container was placed in a mixture solution (100 $\mathrm{mL}$ oil and $500 \mathrm{~mL}$ water). When the pressure of peristaltic pump provided by regulating the voltage of DC supplier was exerted at the end of the pipe, the oil stored in the 3D nickel foam flowed along the pipe into the collector. During the separation, the oil component was constantly supplemented, and the 3D nickel foam could not be submerged by the oil component.

For the oil spill cleanup test, the separation efficiency $(\mathrm{R})$ was calculated with the following equation:

$\mathrm{R}(\%)=(\mathrm{M} / \mathrm{Mo}) \times 100 \%$

where Mo is the original oil mass in the mixture solvent and $\mathrm{M}$ is the collected oil mass after separation process. 
The flux of the oil that permeate through the 3D nickel foam $(F)$ was measured in the equation.

$\mathrm{F}\left(\mathrm{Lm}^{-2} \mathrm{~h}^{-1}\right)=\mathrm{V} /(\mathrm{S} \times \mathrm{T})$

Where $\mathrm{L}$ is the flow volume, $\mathrm{S}$ is the valid flow area of the $3 \mathrm{D}$ nickel foam and $\mathrm{T}$ is the separation time.

\subsection{Characterization}

The hydrophobicity of the nickel foam was measured by an optical contact angle measuring device (JGW-360A, Chengdeshi Shipeng Detection Equipment Co., Ltd) using a droplet (5.0 $\mu \mathrm{L})$ of water or lubricate oil as the indicator at room temperature. The rolling angles were investigated by tilting the nickel foam with a dropler $(5.0 \mu \mathrm{L})$ that came in contact with the nickel foam until the droplet started to slide. The average values were achieved by examining five points on the identical film. The chemical composition of the composite coating was characterized by a Tensor 27 infrared spectrometer (FT-IR). The porous structure of the nickel foam was observed with a scanning electron microscope (Quanta 200, SuZhou Bondray Electronics Technology Co., Ltd). Before observation, the samples were coated with gold using a sputtering coater. The energy-dispersive X-ray spectroscopy (EDS) was taken using an Oxford Instruments X-Max $80 \mathrm{~mm} 2$ silicon drift detector for determining the elemental species of the samples.

\section{Results and discussion}

\subsection{Contact angle}

Fig. 1a shows that FPUH coated nickel foam has a superhydrophobic as well as superoleophilic, behaving like water, acid solution, basic solution, lubricating oil and silicone oil. A drop of water displays quasi-spherical shape with the static WCA of about $157^{\circ} \pm 1^{\circ}$ (Fig. 1b), and the slide angle (SA) reaches a value of about $1^{\circ}$ (Fig. 1d). While a lubricating oil droplet is completely absorbed by 
the surface with the OCA of almost $0^{\circ}$ (Fig. 1c). When the as-prepared foam and the original foam nickel are immersed into water, the original foam nickel is quickly wetted by water (Fig. 1e), while the surface of as-prepared foam exhibits a silver mirror without dampened by water (Fig. 1f).

\subsection{Chemical characterization of the prepared nickel foam}

The available superhydrophobic modification techniques currently require appropriate surface roughness and chemical composition [34]. The surface chemistry of nickel foam can be confirmed by FT-IR analysis. Fig. 2b shows the FT-IR spectrum of FPH coated nickel foam and FPUH coated nickel foam. The peak at 976,840 and $763 \mathrm{~cm}^{-1}$ are generated by the $\alpha$-phase crystals presented in PVDF skeleton. The main absorption peaks at 1216 and $1155 \mathrm{~cm}^{-1}$ are related to the asymmetric stretching vibration and the symmetrical stretching vibration of the $-\mathrm{CF}_{2^{-}}$, and the $1403 \mathrm{~cm}^{-1}$ peak belongs to $\mathrm{C}-\mathrm{H}$ bending vibration in $-\mathrm{CH}_{2}$ group. Make a comparison between the infrared spectra of FPH coated nickel foam and FPUH coated nickel foam, the absorption peak located at $1725 \mathrm{~cm}^{-1}$ is attributed to the effect of UPU skeleton. Compared with pure UPU, PVDF and FEP (Fig. 2a), the resins on the decorated nickel foams display corresponding absorption.

Fig. 3 show evolutive images in the surface morphology of the nickel foam by the scanning electron microscopy (SEM) at different magnifications. SEM images of pristine nickel foam display three-dimensional (3D) interconnected network structure and a smooth surface morphology (Fig. S3). After chemical etching, the skeleton of the nickel foam is relatively rough (Fig. 3a, b). The etched nickel foam has micrometer-scale benzene ringlike grooves and countless uniform distribution of corrosion pit frames. The benzene ring-like grooves and corrosion pits are attributed to the priority of grain boundary and dislocation corrosion [35]. The voids in such rough structures are used to improve the bonding strength between coating and substrate. The static WCA of the 
etched nicked foam is $0^{\circ}$ in virtue of the $-\mathrm{OH}$ formed by etching. SEM images of the FPH coated nickel foam are depicted in Fig. 3 (c, d). They can be seen that the resins have been successfully connected to the skeletons of nickel foam, nevertheless, there are many large bulks and deep grooves on the surface. These can be attributable to the unstable bonding of the fluorine resins and will become a potential threat to its hydrophobic stability. From Fig. 3 (e,f) and Fig. S (4,5), the surface morphology and the thickness of FPUH coated nickel foams are observed. It is worth reminding that with decoration of UPU, the surface morphology of the nickel foam is uniform with about $20 \mu \mathrm{m}$ thickness, providing the important role in forming stable superhydrophobic surface.

During inspecting the cross-section diagram of FPUH coated nickel foam (Fig. 4a), EDS analyses are used to characterize the elements of the medial and lateral sections of the cross-section (Fig. 4b and c). EDS mappings reveal homogeneous dispersion of $\mathrm{Si}$ and $\mathrm{O}$ elements across the medial and lateral surfaces of FPUH coated nickel foam. The presence of $\mathrm{Si}$ and $\mathrm{O}$ elements are ascribed to $\mathrm{SiO}_{2}$, among $\mathrm{O}$ element is also derived from low-concentration UPU. These results confirm $\mathrm{SiO}_{2}$ and UPU are uniformly distributed on the FPUH coated nickel foam. Comparison of the medial and lateral fluorine element composition of FPUH coated nickel foam before and after heat treatment (Fig. S6), the fluorine element in the lateral is significantly increased after heat treatment. Based on principle of minimum energy, the fluorine-contained groups of PVDF and FEP chain segment migrate toward the upper surface and form a fluorine-covered surface during the process of solvent evaporation. Due to the fluorine segments migration, the hydrophobic property of the resin decorated foam nickel is provided with a stable foundation.

Schematic illustration of the separation mechanism of the oil/water separation and the surface structure pattern of FPUH coated nickel foam are shown in Fig. 5. When oil/water mixtures 
permeate through FPUH coated nickel foam, oil spreads over the surface and quickly passes through the surface while the water remains suspending on the oil-wetted surface. Moreover, a waterdroplet contacts the nickel foam surface, it can be easily run off without leaving any ingredient due to the as-prepared foam possesses Cassie-Baxter state. In order to better understanding the synergy of these components, different component coatings were fabricated. Detailed WCAs, OCAs and SAs of the different coatings before and after the two days of oil immersion test are exhibited in the supplementary information (Table 1). The mechanism of fabricating the superhydropholic and superoleophibic coating is as follow: (1) in the process of the fusion, the -NCO of UPU molecular chain can react with the hydroxyl functional groups of the etched nickel foam surface, forming the interface chemical bonding, therefore, the UPU has strong adhesion to the nickel foam. (2) During the heat treatment, fluorine components prefer to migrate to the lateral of the coating, and FEP particles without melting construct analogous micro-textured structure of the coating and lower the surface energy. (3) the $\mathrm{HSiO}_{2}$ particles are homogeneously distributed to provide the nano-structure of the coating and enhance the scouring resistance [36-38].

\subsection{Low-density oil/water separation}

Owing to the superhydrophobicity and superoleophilicity performance of FPUH coated nickel foam, it is potential to be as an ideal candidate for oil collection. Making use of the flexibility of nickel foam, the nickel foam can be randomly folded, interestingly, folding the nickel foam in one direction repeatedly, the foldability of FPUH coated nickel foam can be improved more than twice than that of pure nickel foam, and the wettability of the FPUH coated nickel foam decreases slightly after rigid folding attribute to the destruction of the surface structure (Fig. S7 in the supplementary information). In our case (Fig. 6), the 3D nickel foam is placed in a mixture solution (100 mL oil 
and $500 \mathrm{~mL}$ water). The solution is stirred by a magnetic mixer, which is used to simulate the waves, and the DC supplier applied a voltage of about $5 \mathrm{~V}$. It can been clearly seen that the oil droplets in contact with the 3D foam nickel are quickly being absorbed (Video 1 in the supplementary). The whole collection process is completed within 3 minutes, leaving a fresh and clean water surface. No water is absorbed in the process of separation, and the separation ratio is $99.7 \%$.

To demonstrate the cleanup process of crude oil leakage, the 3D nickel foam is placed on the crude oil leakage (Fig. S8 and Video 2 in the supplementary information). The 3D nickel foam separation system can float on the water that is laid with crude oil $(50 \mathrm{~g})$, after $6 \mathrm{~min}$, the 3D nickel foam almost collects all the crude oil and the crude oil cleanup rate reaches as high as $97.6 \%$.

\subsection{High-density oil/water separation}

The 3D nickel foam also can be utilized to adsorb and reclaim high density organic solvents from water (Fig. 7 and Video 3 in the supplementary information). The $100 \mathrm{~mL}$ chloroform colored with Oil Red and $500 \mathrm{~mL}$ of water are joined into a 1 liter beaker forming the mixed solution. The stainless steel nut is placed in the 3D nickel foam to make the collector submerge in water, and then the gaps of the 3D nickel foam are filled with the silicone sealant. The FPUH coated nickel foam box allows the chloroform to sheet over the surface while against water. The underwater chloroform solution is completely adsorbed by 3D nickel foam within 70 seconds, and the chloroform cleanup rate reaches $99.8 \%$.

Comparing with recently developed oil absorbent technology that can not directly deal with oil spill pollution in marine or continuously treatment of sewage for a long time, our 3D nickel foam shows high efficiency of the continuous separation of oil removal and collection. In addition, other various porous frameworks of stainless steel meshes and fabrics are also chosen to obtain 
superhydrophobic surfaces (Fig. S9 and Table 2 in the supplementary). The results indicate that they all had good performance, the FPUH coated nickel foam is most stable among the three materials.

\subsection{7 days of continuous separation and durability test}

To evaluate the hydrophobic stability properties of 3D nickel foams with different UPU loading, we measured the static water CAs on various substrates with different separation time. As seen in Figure 8a and Figure S10, the static water CAs decrease with the increase of separation time. The sprayed nickel foam without UPU shows unstable hydrophobicity. After two days of continuous separation, the static water CAs dropped from $153.6^{\circ}$ to $122.3^{\circ}$. The effect get better by increasing UPU content, demonstrating the crucial role of UPU in immobilizing fluorine resin on nickel foam. Obviously, the 3D nickel foam at $10 \mathrm{wt} \%$ UPU concentration gives the highest water CA of $157^{\circ}$, and the $\mathrm{CA}$ of the nickel foam is still larger than $130^{\circ}$ after 7 days absorption, indicating good hydrophobic nature. As the UPU loading increased to $15 \mathrm{wt} \%$, the hydrophobicity of the coating decreased relatively attributing to reduction of fluorine content and the smoothing effect of UPU.

Due to the unavoidable harsh environments, the resistance against corrosion of the 3D nickel foams with different UPU content was investigated by immersing into different solutions for periods of 7 days ( $\mathrm{pH}$ values in the range from 1 to 14 with $10 \mathrm{wt} \% \mathrm{NaCl}$ ). As shown in Fig. 8b, all the static CAs remain above $130^{\circ}$, among alkali resistant capacity is better than acid resistant capacity. The durability and alkali resistant are related to the intrinsic chemical inertness of PVDF and FEP. The solvent resistant ability of the $10 \mathrm{wt} \%$ UPU is more excellent compare to other contents of the nickel foams. Simultaneously, when the 3D nickel foam with $10 \mathrm{wt} \%$ UPU is exposed in $0{ }^{\circ} \mathrm{C}, 60{ }^{\circ} \mathrm{C}$ and ultraviolet radiation environment conditions for 7 days (Fig. S11), the hydrophobicity is the stability, which exhibits the feasibility of a high real-world application. 
Additionally, apart from the hydrophobicity durability and resistance against corrosion, the 3D nickel foam also can absorb a variety of solvents with high oil mass flux and behave high separation efficiency during 7 days separation. In our works, the oils and organic solvents were used including lubricating oil, silicon oil, petroleum ether, corn oil, dichloromethane, chloroform and toluene. As illustrated in Figure 8c, the 3D nickel foam exhibits fluxes increase ranging from $1744 \mathrm{Lm}^{-2} \mathrm{~h}^{-1}$ to $5460 \mathrm{Lm}^{-2} \mathrm{~h}^{-1}$ with decreasing the densities and viscosities of absorbed solvents during 7 days absorption. The as-prepared 3D nickel foam is capable of absorbing lubricating oil up to $204 \mathrm{~L}$ with high separation efficiency after 7 days separation (Fig. S12 in the supplementary information). As detailed in Fig. 8d and Fig. S13, the separation efficiency remain above $99.0 \%$ after 7 days separation, and no obvious water is absorbed, exhibiting its great potential in practical application. As shown in Table 1, in terms of the durability and separation effciency, the property of our 3D nickel foam outshines the reported ones with longer separation time and higher effciency.

\subsection{The Recycled Experiments of Oil/Water Separation}

After 7 days in oil-water separation, we got the nickel foam box out of the beaker and put it at room temperature for one day. Then, the nickel foam box was washed with warm water three times and dried in a $80{ }^{\circ} \mathrm{C}$ oven. Due to the constant scour of the liquid, the loss of the resins on the skeleton are inevitable during the oil-water separation. As displayed in Fig. 9a and b, there are still a large amount of resins and nano structure on the surface. After that, the $3 \mathrm{D}$ nickel foam was put into an oven at $240{ }^{\circ} \mathrm{C}$ to burn for 2 hours. Surprisingly, the WCA of 3D nickel foam can be restored to $150^{\circ}$ (Fig. 9c and d). During the heat treatment, the hydrophobicity on the surface is recovered on account of the rearrangement of the chains in thermoplastic fluorine material that minimizes the surface free energy after the oil evaporating. As shown in Fig. S14, the weight of the PFUH coated 
nickel foam decreased obviously at $450{ }^{\circ} \mathrm{C}$ under the condition of nitrogen, verifying the coating possesses good thermal stability.

To better evaluate the recyclability performance of the 3D nickel foam, we carried out one more oil-water separation test. The separation time was $1 \mathrm{~h}$ and the volume of oil solution passing through the 3D nickel foam was about $1900-2100 \mathrm{~mL}$ in each cycle. The static WCAs record for 3D nickel foam after every cycle (Fig. 10a). As separation time goes on, the static WCAs decrease slightly from $150.2^{\circ}$ to $143.1^{\circ}$, the SAs increase from $1^{\circ}$ to $9.8^{\circ}$. Fig. $10 \mathrm{~b}$ exhibits the change of WCA after repeating immerse/heat cycles (immerse into oil solution 24 hours in each cycle). Overall, the hydrophobicity recovery capability of FPUH surface is improved by this simple heating treatment.

\section{Conclusion}

In summary, the superhydrophobic and superoleophilic FPUH coated nickel foam was successfully fabricated, controlling external and internal nano/micro-structure simultaneously, by using a one-step spraying technology. Furthermore, the device for continuous removal and absorption oil or organic solution from water surface could be designed by the foldable nickel foam into a functional box. After 7 days continuous oil/water separation and immersion in different strong solutions, we discovered that the increasing addition of UPU could enhance the superhydrophobic stability of the 3D nickel foam. The 3D nickel foam with $10 \mathrm{wt} \%$ UPU could achieved the best superhydrophobicity and superoleophilicity, as well as stability. Finally, after a long time of oil-water separation, the surface morphology of the box could be recovered by $240{ }^{\circ} \mathrm{C}$ heat treatment, with a static WCA of $150^{\circ}$ to extend the using time. Because of its facile preparation, continuous separation and superhydrophobic durability, this 3D nickel foam will be of great potential for the oil-water separation. 


\section{Acknowledgements}

The research is financially supported by the National Young Top Talents Plan of China (2013042), National Science Foundation of China $(21676052$, 21606042), the Science Foundation for Distinguished Young Scholars of Heilongjiang Province (JC201403).

\section{Notes and references}

[1] Z. Chu, Y. Feng and S. Seeger, Oil/Water separation with selective superantiwetting / superwetting surface materials, Angew. Chem. Int. Ed. 54 (2015) 2328-2338.

[2] C. Su, H. Yang, S. Song, B. Lu and R. Chen, A magnetic superhydrophilic / oleophobic sponge for continuous oil-water separation, Chemical Engineering Journal 309 (2017) 366-373.

[3] C. Zhou, J. Cheng, K. Hou, A. Zhao, P. Pi, X. Wen and S. Xu, Superhydrophilic and underwater superoleophobic titania nanowires surface for oil repellency and oil/water separation, Chemical Engineering Journal 301 (2016) 249-256.

[4] K. Gaaseidnes and J. Turbeville, Separation of oil and water in oil spill recovery operations, Pure Appl. Chem. 71 (1999) 95-101. 
[5] V. Broje and A.A. Keller, Improved mechanical oil spill recovery using an optimized geometry for the skimmer surface, Environ. Sci. Technol. 40 (2006) 7914-7918.

[6] I. Buist, J. McCourt, S. Potter, S. Ross and K. Trudel, In situ burning, Pure and Applied Chemistry 71 (1999) 43-65.

[7] L. Wen, Y. Tian and L. Jiang, Bioinspired super-wettability from fundamental research to practical applications, Angew. Chem. Int. Ed. 54 (2015) 3387-3399.

[8] B. Su, Y. Tian and L. Jiang, Bioinspired interfaces with superwettability: From materials to chemistry, J. Am. Chem. Soc. 138 (2016) 1727-1748.

[9] J. Song, Y. Lu, J. Luo, S. Huang, L. Wang, W. Xu and I. P. Parkin, Barrel-shaped oil skimmer designed for collection of oil from spills, Adv. Mater. Interfaces 2 (2015) 1500350.

[10] Q. Zhu, Y. Chu, Z. Wang, N. Chen, L. Lin, F. Liu and Q. Pan, Robust superhydrophobic polyurethane sponge as a highly reusable oil-absorption material, J. Mater. Chem. A 1 (2013) 5386-5393.

[11] X. Zhou, Z. Zhang, X. Xu, F. Guo, X. Zhu, X. Men and B. Ge, Robust and durable superhydrophobic cotton fabrics for oil/water separation, ACS Appl. Mater. Interfaces 5 (2013) 7208-7214.

[12] J. Zhang and S. Seeger, Polyester materials with superwetting silicone nanofilaments for oil/water separation and selective oil absorption, Adv. Funct. Mater. 21 (2011) 4699-4704.

[13] C.R. Crick, J.A. Gibbins and I.P. Parkin, Superhydrophobic polymer-coated copper-mesh; membranes for highly efficient oil-water separation, J. Mater. Chem. A 1 (2013) 5943-5948. 
[14] B. Wang and Z. Guo, Superhydrophobic copper mesh films with rapid oil/water separation properties by electrochemical deposition inspired from butterfly wing, Applied Physics Letters 103 (2013) 063704.

[15] S. Wang, Y. Song, L. Jiang, Microscale and nanoscale hierarchical structured mesh films with superhydrophobic and superoleophilic properties induced by long-chain fatty acids, Nanotechnology 18 (2007) 15103.

[16] C. Su, Z. Lu, H. Zhao, H. Yang and R. Chen, Photoinduced switchable wettability of bismuth coating with hierarchical dendritic structure between superhydrophobicity and superhydrophilicity, Applied Surface Science 353 (2015) 735-743.

[17] E. Wang, H. Wang, Z. Liu, R. Yuan and Y. Zhu, One-step fabrication of a nickel foam-based superhydrophobic and superoleophilic box for continuous oil-water separation, J Mater Sci 50 (2015) 4707-4716.

[18] Z. Wang, Y. Xu, Y. Liu and L. Shao, A novel mussel-inspired strategy toward superhydrophobic surfaces for self-driven crude oil spill cleanup, J. Mater. Chem. A 3 (2015) 12171-121718.

[19] X. Gao, J. Zhou, R. Du, Z. Xie, S. Deng, R. Liu, Z. Liu and J. Zhang, Robust superhydrophobic foam: A graphdiyne-based hierarchical architecture for oil/water separation, Adv. Mater. 28 (2016) 168-173.

[20] J. Li, L. Yan, X. Tang, H. Feng, D. Hu and F. Zha, Robust Superhydrophobic Fabric Bag Filled with Polyurethane Sponges Used for Vacuum-Assisted Continuous and Ultrafast Absorption and Collection of Oils from Water, Adv. Mater. Interfaces 3 (2016) 1500770-1500777. 
[21] S. Song, H. Yang, C. Su, Z. Jiang and Z. Lu, Ultrasonic-microwave assisted synthesis of stable reduced graphene oxide modified melamine foam with superhydrophobicity and high oil adsorption capacities, Chemical Engineering Journal 306 (2016) 504-511.

[22] M.W. Lee, S. An, S.S. Latthe, C. Lee, S. Hong and S.S. Yoon, Electrospun polystyrene nanofiber membrane with superhydrophobicity and superoleophilicity for selective separation of water and low viscous oil, ACS applied materials \& interfaces 5 (2013) 10597-10604.

[23] M. Obaid, N.A.M. Barakat, O.A. Fadali, M. Motlak, A.A. Almajid, K.A. Khalil, Effective and reusable oil/water separation membranes based on modified polysulfone electrospun nanofiber mats, Chemical Engineering Journal 259 (2015) 449-456.

[24] J. Li, L. Yan, H. Li, J. Li, F. Zha and Z. Lei, A facile one-step spray-coating process for the fabrication of a superhydrophobic attapulgite coated mesh for use in oil/water separation, RSC Advances 5 (2015) 53802-53808.

[25] K. Sasaki, M. Tenjimbayashi, K. Manabe and S. Shiratori, Asymmetric superhydrophobic / superhydrophilic cotton fabrics designed by spraying polymer and nanoparticles, ACS Appl. Mater. Interfaces 8 (2016) 651-659.

[26] M.A. Gondal, M.S. Sadullah, M.A. Dastageer, G.H. McKinley, D. Panchanathan and K.K. Varanasi, Study of factors governing oil-water separation process using $\mathrm{TiO}_{2}$ films prepared by spray deposition of nanoparticle dispersions, ACS Appl. Mater. Interfaces 6 (2014) 13422-13429.

[27] J. Yang, L. Yin, H. Tang, H. Song, X. Gao, K. Liang and C. Li, Polyelectrolyte-fluorosurfactant complex-based meshes with superhydrophilicity and superoleophobicity for oil/water separation, Chemical Engineering Journal 268 (2015) 245-250. 
[28] H. Wang, E. Wang, Z. Liu, D. Gao, R. Yuan, L. Sun and Y. Zhu, A novel carbon nanotubes reinforced superhydrophobic and superoleophilic polyurethane sponge for selective oil-water separation through a chemical fabrication, J. Mater. Chem. A 3 (2015) 266-273.

[29] J. Li, C. Xu, Y. Zhang, J. Li, C. Xu, Y. Zhang, R. Wang, F. Zha and H. She, Robust superhydrophobic attapulgite coated polyurethane sponge for efficient immiscible oil/water mixture and emulsion separation, Journal of Materials Chemistry A 4 (2016) 15546-15553.

[30] N. Cao, B, Yang, A. Barras and N. Yang, Polyurethane sponge functionalized with superhydrophobic nanodiamond particles for efficient oil/water separation, Chemical Engineering Journal 307 (2017) 319-325.

[31] S. Zhou, G. Hao, X. Zhou, W. Jiang, T. Wang, N. Zhang and L. Yu, One-pot synthesis of robust superhydrophobic, functionalized graphene/polyurethane sponge for effective continuous oil-water separation, Chemical Engineering Journal 302 (2016) 155-162.

[32] X. Zhang, D. Zhi, W. Zhu, S. Sathasivamb and I. Parkin, Facile fabrication of durable superhydrophobic $\mathrm{SiO}_{2}$ /polyurethane composite sponge forcontinuous separation of oil from water, RSC Adv. 7 (2017) 11362-11366.

[33] B. Wang, W. Liang, Z. Guo and W. Liu, Biomimetic super-lyophobic and super-lyophilic materials applied for oil/water separation: a new strategy beyond nature, Chem. Soc. Rev. 44 (2015) 336-361.

[34] J. Ge, Y. Ye, H. Yao, X. Zhu, X. Wang, L. Wu, J. Wang, H. Ding, N. Yong, L. He and S. Yu, Pumping through porous hydrophobic/oleophilic materials: An alternative technology for oil spill remediation, Angew. Chem. 126 (2014) 3686-3690. 
[35] B. Qian and Z. Shen, Fabrication of superhydrophobic surfaces by dislocation-selective chemical etching on aluminum, copper, and zinc substrates, Langmuir 21 (2005) 9007-9009.

[36] H. Yang, P. Pi, Z. Yang, Z. Lu and R. Chen, Design of a superhydrophobic and superoleophilic film using curedfluoropolymer@ silica hybrid, Applied Surface Science 388 (2016) 268-273.

[37] H. Yang, X. Hu, R. Chen, S. Liu, P. Pi and Z. Yang, Fluoropolymer/SiO $\mathrm{S}_{2}$ composite films with switchable superoleophilicity and high oleophobicity for"on-off"oil permeation, Applied Surface Science 280 (2013) 113-116.

[38] X. Zeng, L. Qian, X. Yuan, C. Zhou, Z. Li, J. Cheng, S. Xu, S. Wang, P. Pi and X. Wen, Inspired by Stenocara Beetles: From Water Collection to High-Efficiency Water-in-Oil Emulsion Separation, ACS Nano 11 (2017) 760-769.

[39] X. Jin, B. Shi, L. Zheng, X. Pei, X. Zhang, Z. Sun, Y. Du, J. Kim, X. Wang, S. Dou, K. Liu and L. Jiang, Bio-inspired multifunctional metallic foams through the fusion of different biological solutions, Advanced Functional Materials 24 (2014) 2721-2726.

[40] C. Xue, Y. Li, J. Hou, L. Zhang, J. Ma and S. Jia, Self-roughened superhydrophobic coatings for continuous oil-water separation, J. Mater. Chem. A 3 (2015) 10248-10253. 


\section{Table}

Table 1 The comparisons of different absorbent materials

\begin{tabular}{|c|c|c|c|c|c|}
\hline $\begin{array}{c}\text { Oil/water separation } \\
\text { materials }\end{array}$ & $\begin{array}{l}\text { Preparation } \\
\text { methods }\end{array}$ & $\begin{array}{l}\text { Separation } \\
\text { substances }\end{array}$ & $\begin{array}{l}\text { Separation } \\
\text { condition }\end{array}$ & $\begin{array}{c}\text { Separation } \\
\text { effciency } \\
(\%)\end{array}$ & Reference \\
\hline ODA/FA/fabric & $\begin{array}{l}\text { in-situ } \\
\text { synthesis }\end{array}$ & crude oil & $\begin{array}{l}15 \mathrm{~g} \text { crude } \\
\text { oil }\end{array}$ & 97.1 & [18] \\
\hline $\begin{array}{l}\text { trimethoxysilane/graphite } \\
\text { oxide/PU sponge }\end{array}$ & dip coating & lubricating oil & $15 \mathrm{~L}$ & 99.5 & {$[31]$} \\
\hline $\begin{array}{l}\text { thiolated graphene/PU } \\
\text { sponge }\end{array}$ & dip coating & toluene & 5 cycles & 99.5 & [39] \\
\hline $\begin{array}{l}\text { Polydimethylsiloxane/poly } \\
\text { vinyl chloride/PET textile }\end{array}$ & $\begin{array}{c}\text { phase-separ } \\
\text { ation }\end{array}$ & diesel oil & $9 \mathrm{~h}$ & 97 & {$[40]$} \\
\hline \multirow[t]{3}{*}{ FPUH coated nickel foams } & spraying & crude oil & $\begin{array}{l}50 \mathrm{~g} \text { crude } \\
\text { oil }\end{array}$ & 97.6 & \\
\hline & & lubricating oil & 7 days & 99.6 & \\
\hline & & chloroform & 7 days & 99.8 & Our work \\
\hline
\end{tabular}




\section{Figures}

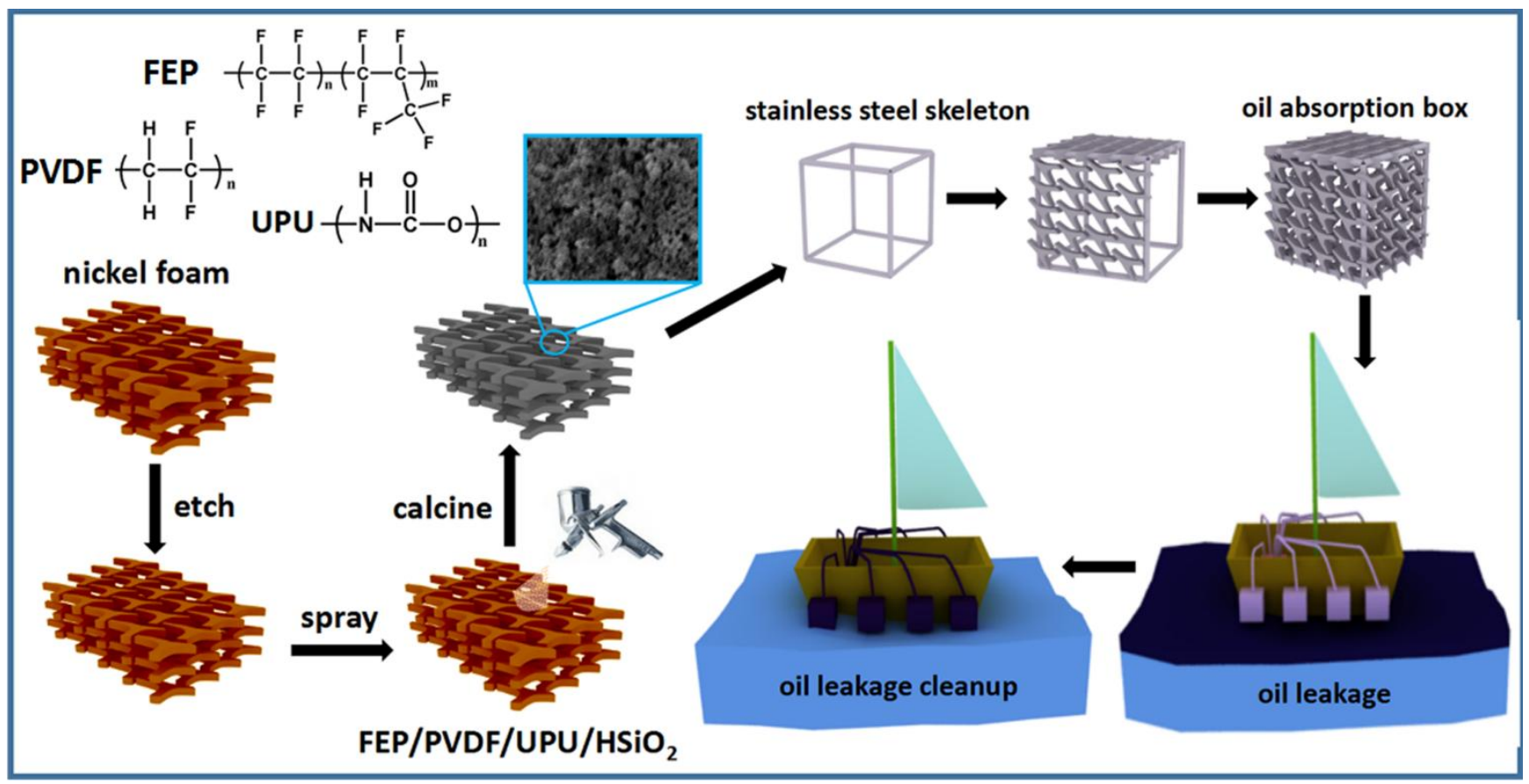

Scheme 1 Schematic drawing of the fabrication of superhydrophobic and superoleophilic nickel foam. 

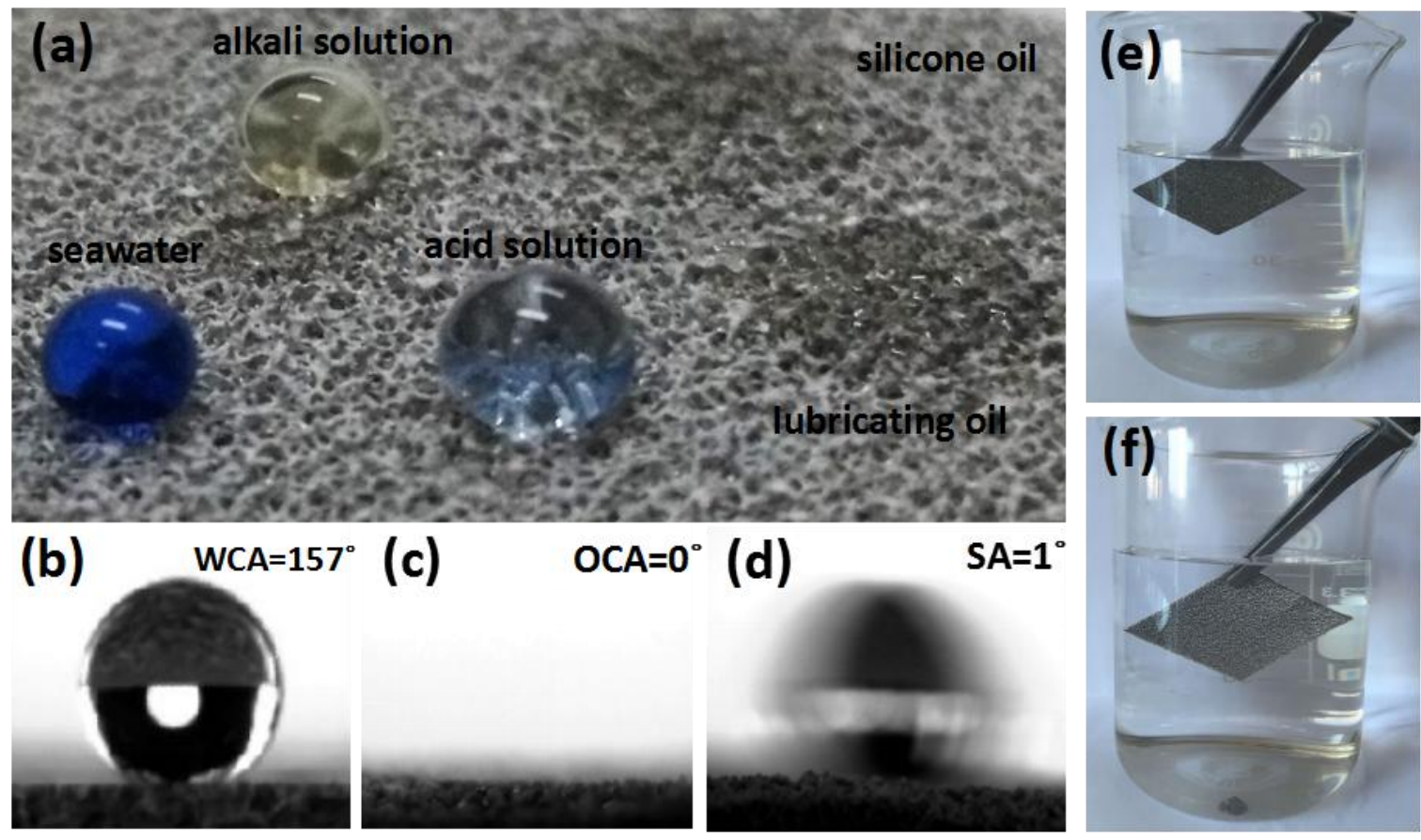

Fig.1. Digital photographs of different solution droplets on the FPUH coated nickel foam (a) WCA
of $157^{\circ}$
(b) OCA of $0^{\circ}$
(c) and SA of $1^{\circ}$
(d) on the the FPUH coated nickel foam. Picture of pure

nickel foam (e) and FPUH coated nickel foam (f) are immersed into water under external force.
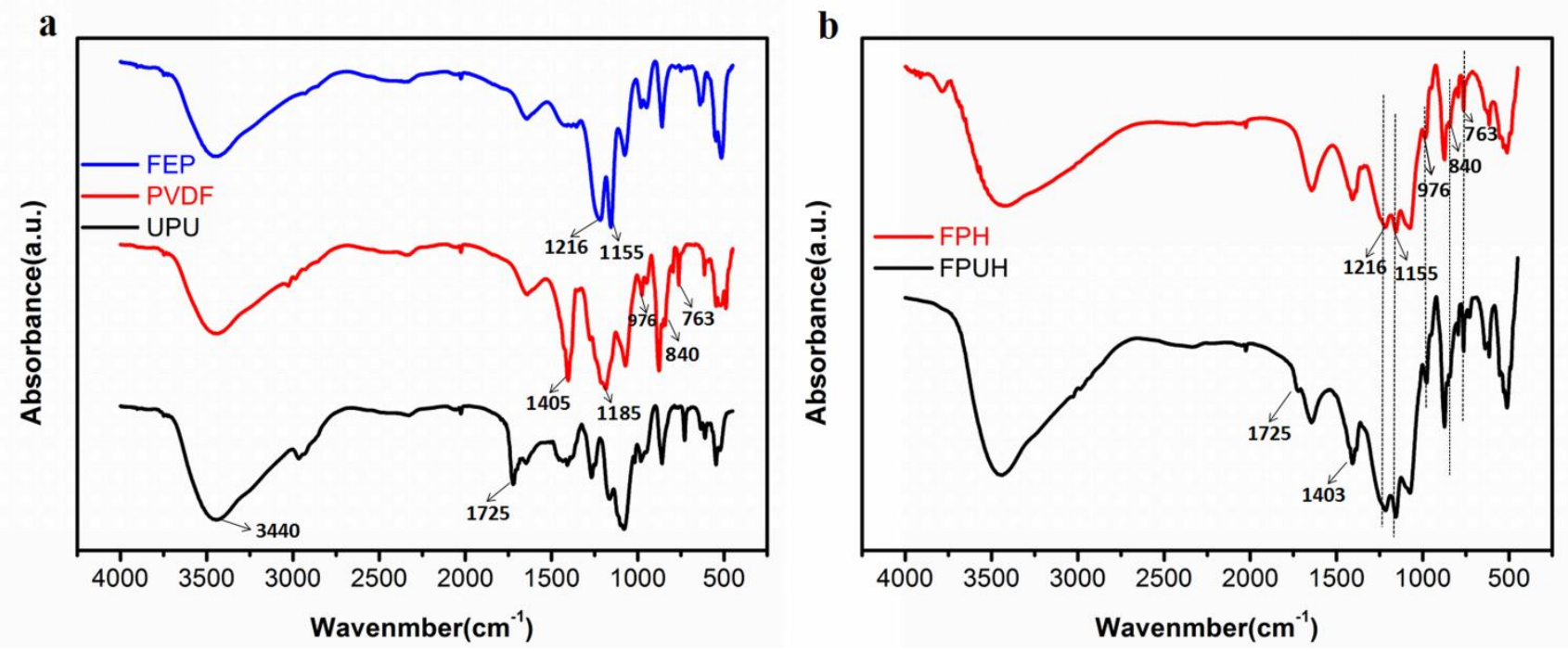

Fig.2. FT-IR spectra of the FEP, PVDF and UPU (a), and the FPH and the FPUH (b) composite nickel foams. 

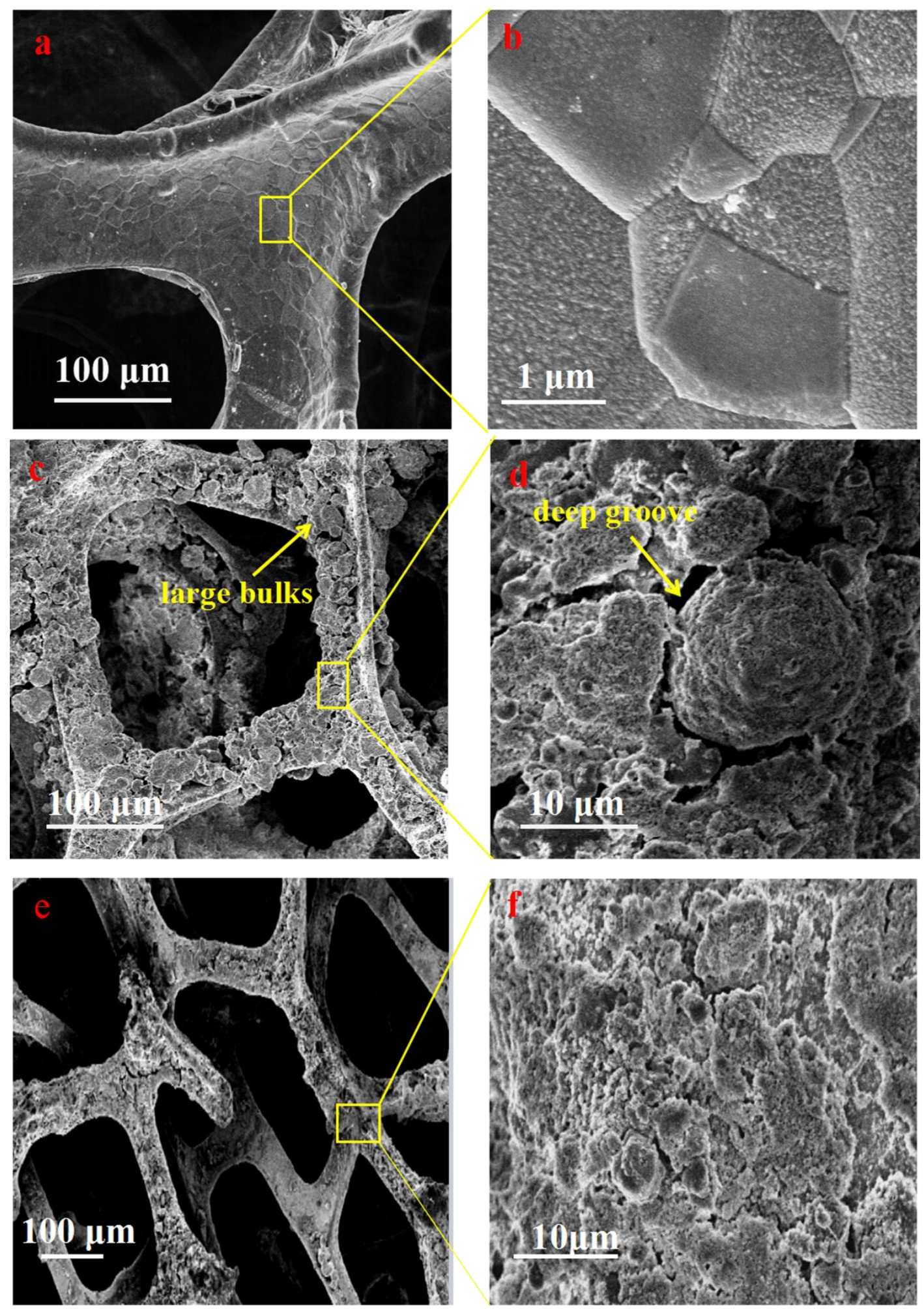

Fig.3. SEM images: $a$ and $b$ etched nickel foam, $c$ and d FPH coated nickel foam, e and f FPUH coated nickel foam. 


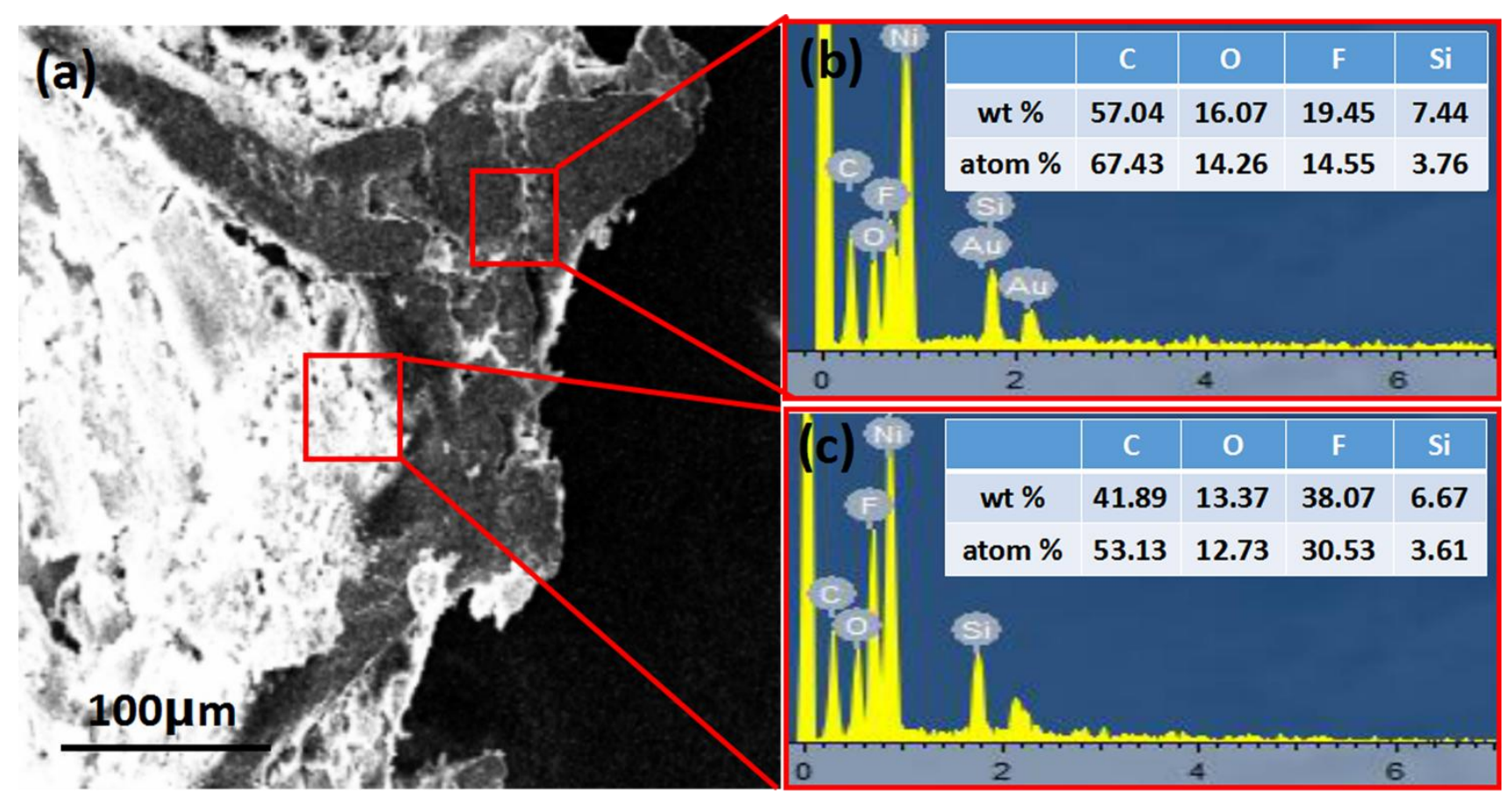

Fig.4. SEM image of cross-section diagram of FPUH coated nickel foam (a) EDS mapping of chemical composition analysis of the cross-section of the medial diagram (b) and the cross-section of the lateral diagram (c) of FPUH coated nickel foam. 


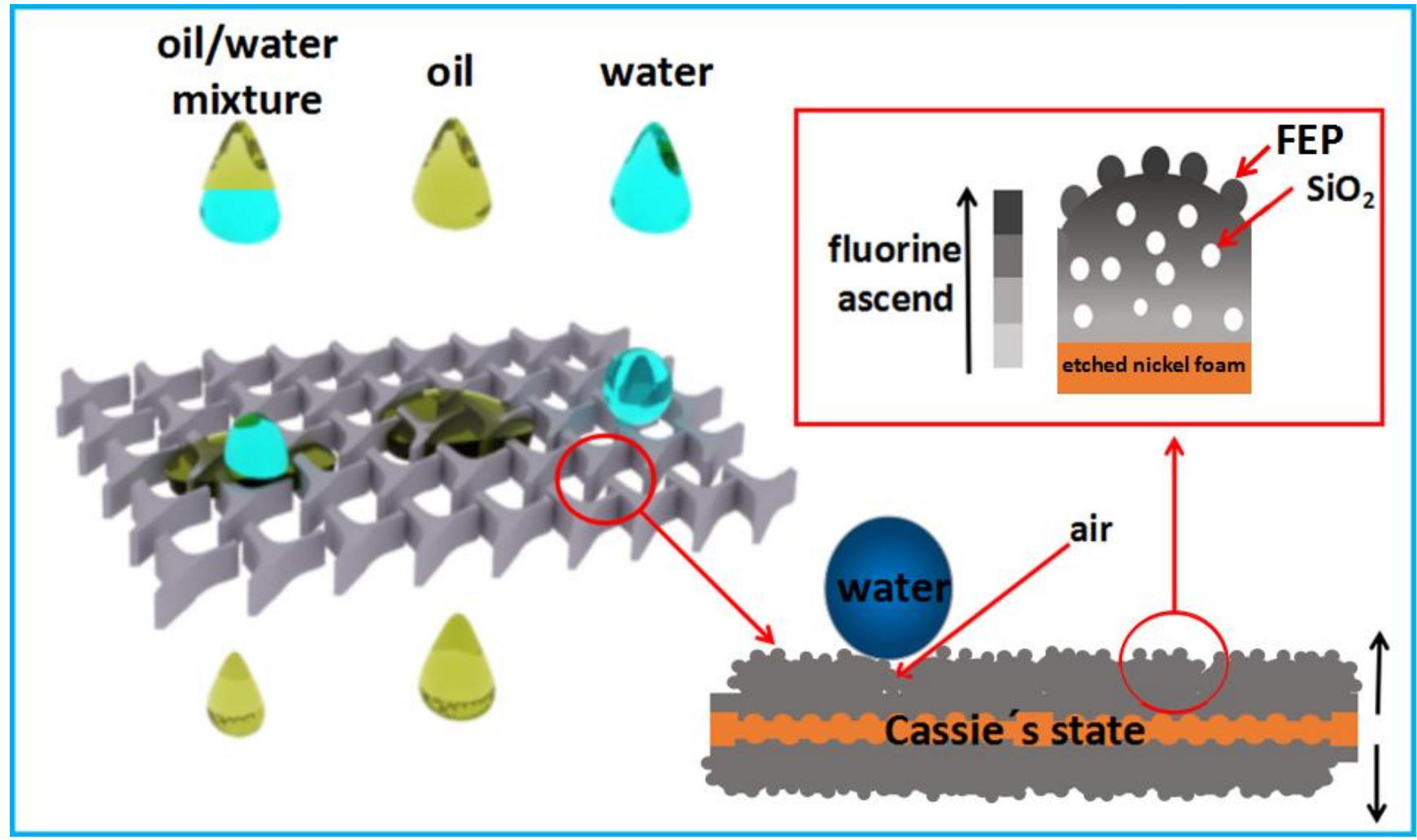

Fig.5. The separation mechanism of the oil/water separation and the surface microstructure pattern of FPUH coated nickel foam. 


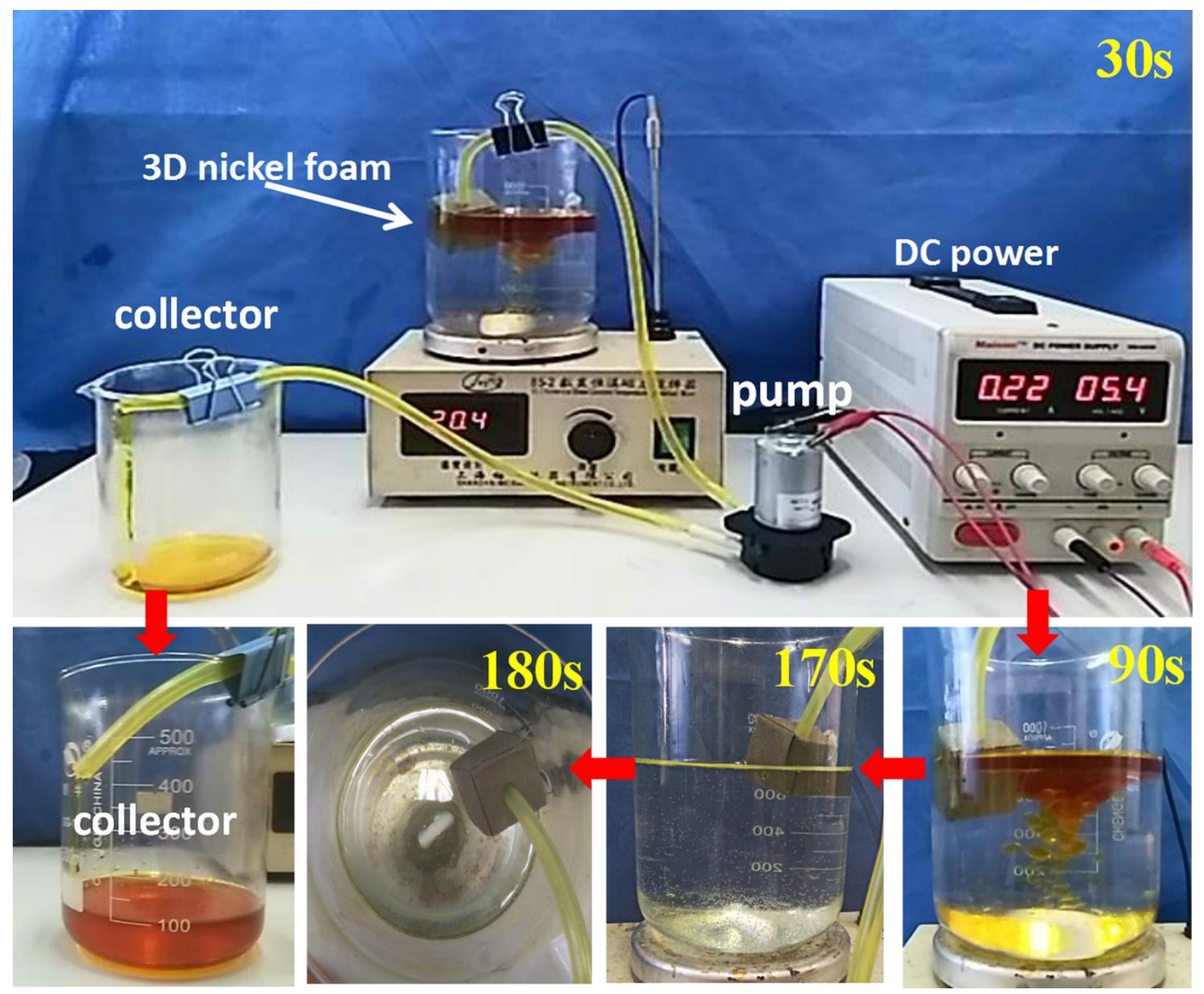

Fig.6. Photographs of continuous collection lubricating oil in situ from water with the 3D nickel foam.
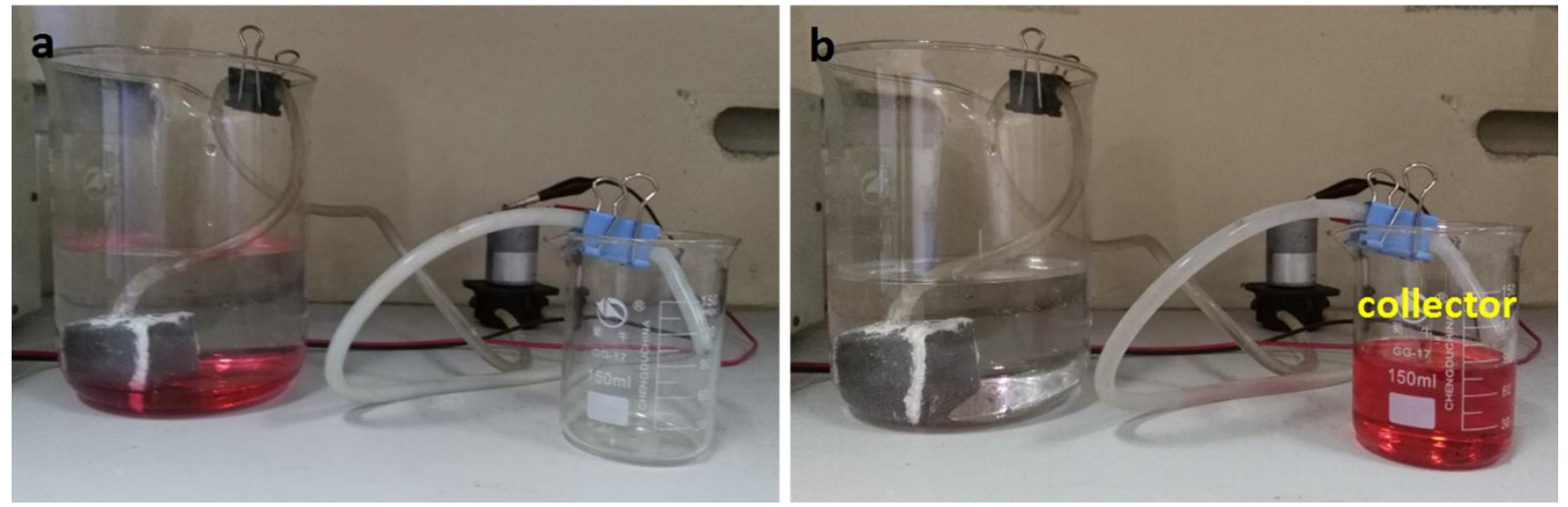

Fig.7. Photographs of continuous collection choroform in situ from water with the 3D nickel foam. 

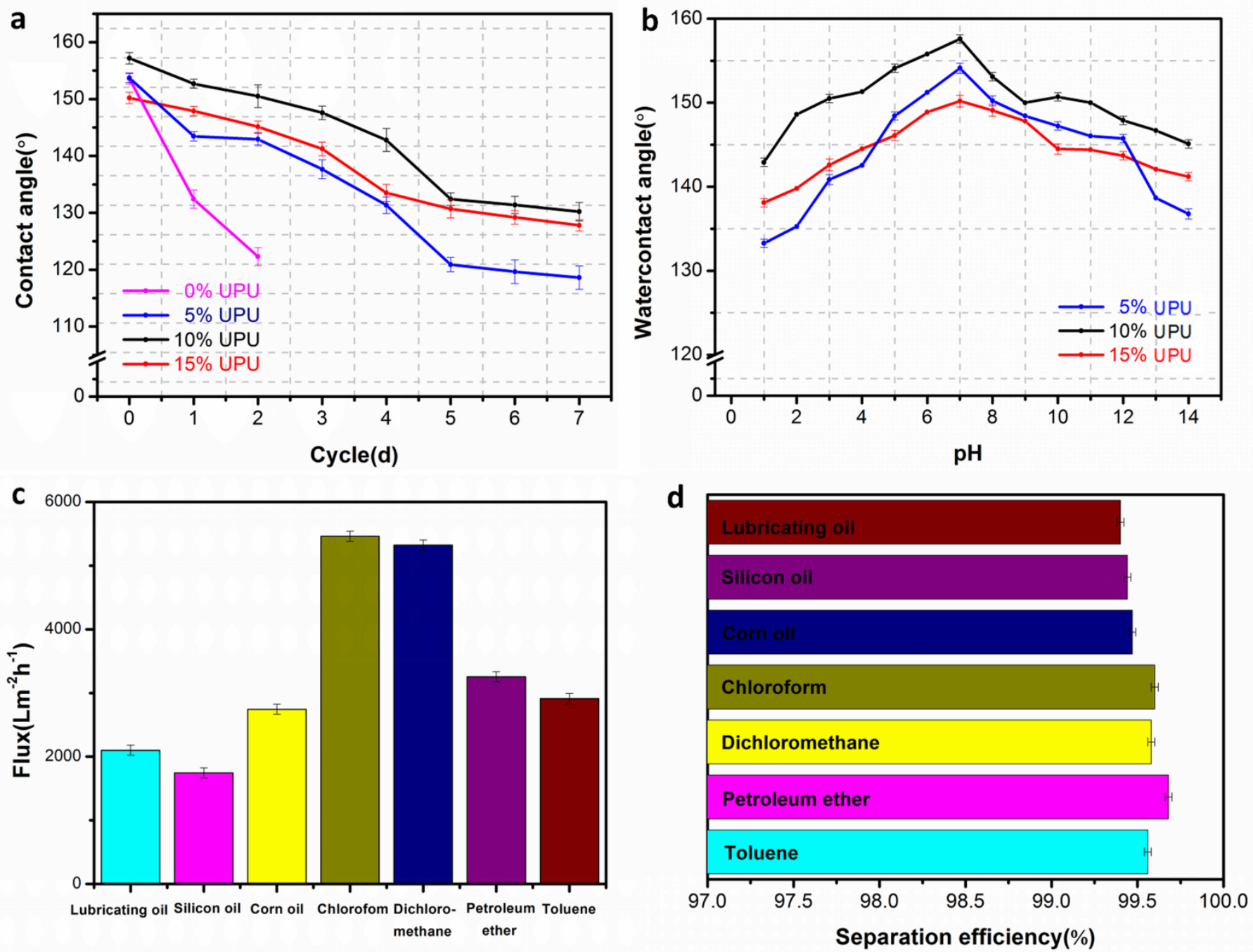

Fig.8. The static WCAs recorded for 3D nickel foam with different UPU loading (a) after each oil/water separation and (b) after immersion into different solutions for 7 days. (c) The absorption flux and (d) the separation efficuency of 3D nickel foam for common organic solvents during 7 days separation. 

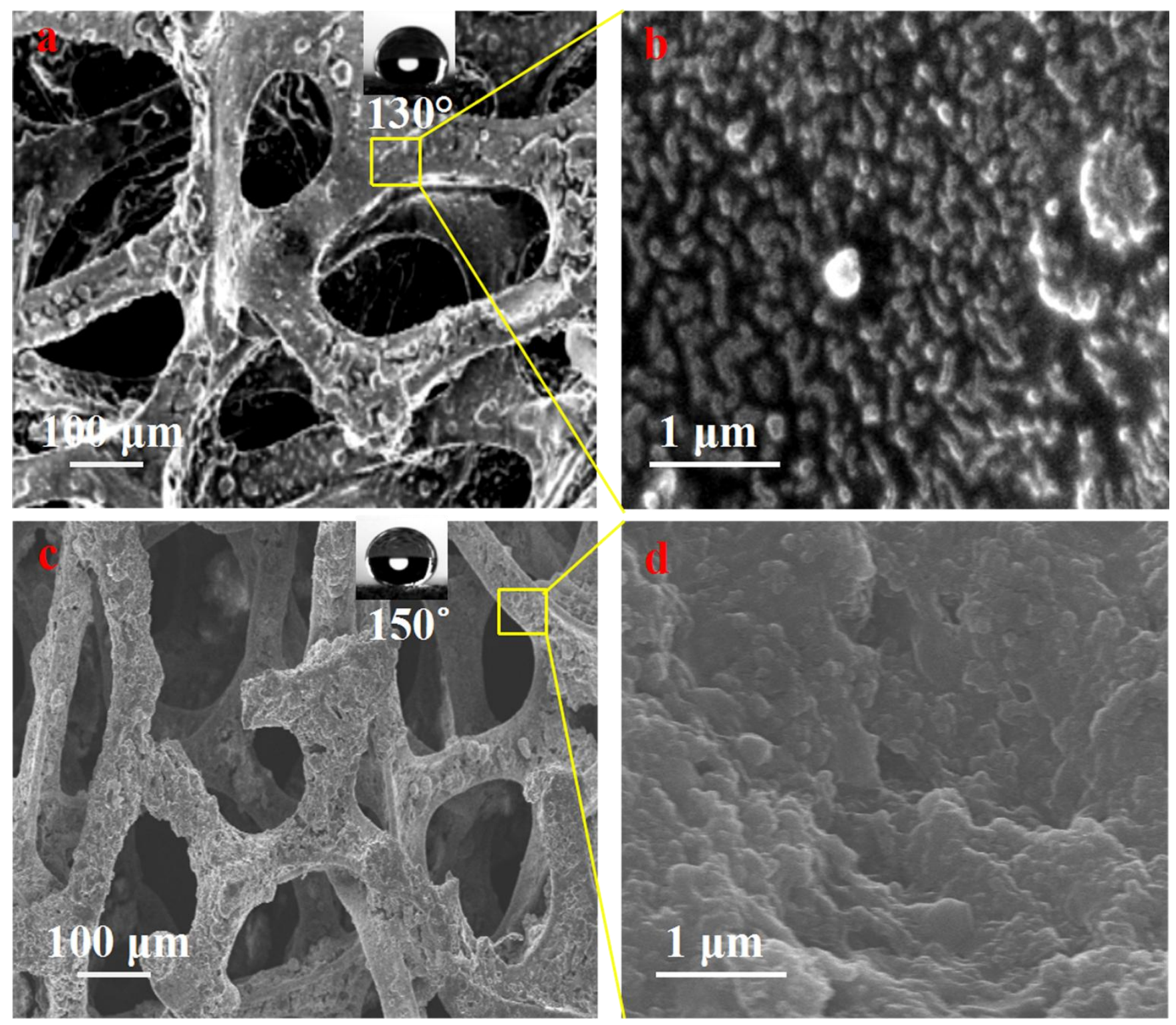

Fig.9. SEM images of the nicked foams after wash ( $a$ and $b$ ), and after heat treatment (c and d). 

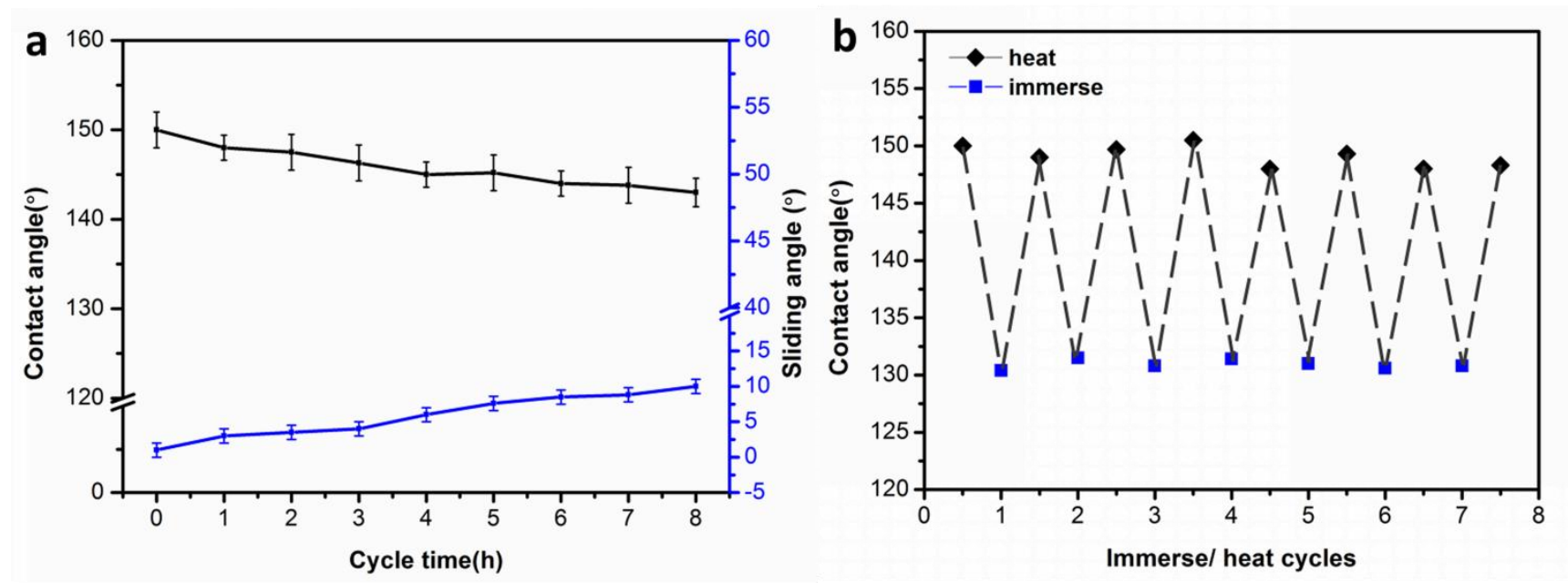

Fig.10. The static WCAs and SAs recorded for 3D nickel foam after cycle (a) The WCA after repeating immerse/heat cycles (b). 


\section{Graphical abstract}

Facile preparation of superhydrophobic metal foam for durable and high

\section{efficient continuous oil-water separation}

Yue Hu, Yanji Zhu, Huaiyuan Wang*, Chijia Wang, Hongwei Li, Xiguang Zhang, Ruixia Yuan, Yiming Zhao

College of Chemistry and Chemical Engineering, Northeast Petroleum University, Daqing, China

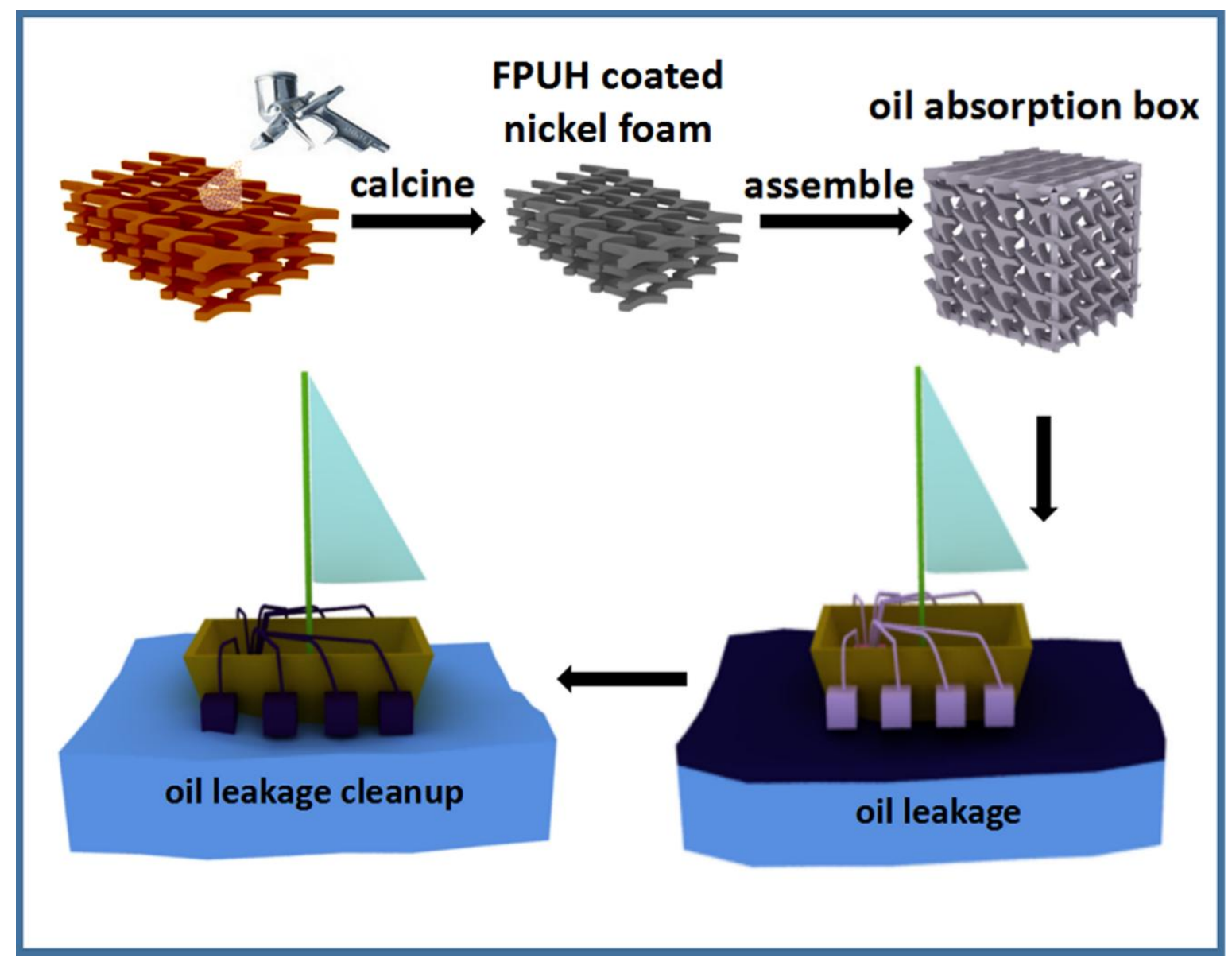

"Corresponding author. Tel.: 0086-4596503083; Fax: 0086-4596503083.

E-mail address: wanghyjiji@163.com (H.Y.Wang) 